\title{
Concise report
}

\section{Association of $H L A-D R B 1$ shared epitope alleles and immune checkpoint inhibitor-induced inflammatory arthritis}

\author{
Laura C. Cappelli ${ }^{1}$, Mehmet T. Dorak ${ }^{2}$, Maria P. Bettinotti ${ }^{3}$, \\ Clifton O. Bingham III ${ }^{1}$ and Ami A. Shah ${ }^{1}$
}

\begin{abstract}
Objective. To evaluate the frequency of HLA class I and II alleles associated with traditional forms of inflammatory arthritis in patients with immune checkpoint inhibitor (ICl)-induced inflammatory arthritis as compared with population controls.

Methods. High-resolution HLA typing was performed on 27 patients with ICl-induced inflammatory arthritis and 726 healthy controls. Genotyping at the shared epitope (SE) locus (HLA DRB1) was performed on 220 RA cases. Allele-positivity rates and frequency of having at least one SE allele were compared using Fisher's exact test between $\mathrm{ICl}$-induced inflammatory arthritis and healthy controls. Frequency of having at least one SE allele was also compared between ICl-induced inflammatory arthritis and RA cases.

Results. Twenty-six patients with ICl-induced inflammatory arthritis were of European descent, and one was African American. In those 26 patients, $16(61.5 \%)$ had at least one SE allele, significantly different from healthy controls of European descent, in whom $299(41.2 \%)$ had at least one SE allele (odds ratio 2.3, $P=0.04)$. The allele-positivity rate of DRB1*04: 05 was also higher in the $\mathrm{ICl}$-induced inflammatory arthritis group. The $\mathrm{ICl}$-induced inflammatory arthritis population and RA patients of European descent did not differ in frequency of having at least one SE allele, but $\mathrm{ICl}$-induced inflammatory arthritis patients were more likely to be autoantibody-negative for RF and anti-CCP antibodies. Conclusion. Patients with ICl-induced inflammatory arthritis of European descent were more likely to have at least one SE allele than healthy controls. Further studies are needed to validate these findings and investigate whether a unique immunogenetic framework increases risk for different immune-related adverse events.
\end{abstract}

Key words: inflammatory arthritis, cancer immunotherapy, immune checkpoint inhibitors, immunogenetics

\section{Rheumatology key messages}

- Shared epitope alleles were more common in immune checkpoint inhibitor-induced inflammatory arthritis than in population controls.

- Checkpoint inhibitor-induced inflammatory arthritis and RA populations had similar frequencies of shared epitope alleles.

- A patient's immunogenetic framework may influence the development of immune-related adverse events.

\section{Introduction}

Class I and II HLA alleles are important genetic risk factors for a variety of autoimmune diseases [1]. Associations

${ }^{1}$ Division of Rheumatology, The Johns Hopkins University School of Medicine, Baltimore, MD, USA, ${ }^{2}$ School of Life Sciences, Pharmacy \& Chemistry, Kingston University London, London, UK and ${ }^{3}$ Immunogenetics Laboratory, Department of Medicine, The Johns Hopkins University School of Medicine, Baltimore, MD, USA Submitted 19 July 2018; accepted 30 September 2018

Correspondence to: Laura C. Cappelli, Department of Medicine, Division of Rheumatology, Johns Hopkins University School of Medicine, 5501 Hopkins Bayview Circle, Suite 1B1, Baltimore, MD 21224, USA.

E-mail: Icappel1@jhmi.edu with particular HLA alleles have informed models of pathogenesis for autoimmunity, such as in RA, where increased presentation of citrullinated antigens by HLA class II shared epitope (SE) is hypothesized as relevant to underlying immune biology [2].

Immune-related adverse events (irAEs) from immune checkpoint inhibitor $(\mathrm{ICl})$ therapy can be considered novel immune mediated diseases. ICls, used in the treatment of a variety of malignancies, block negative costimulatory molecules, leading to increased immune activation [3]. As a result of activation of $T$ cells and downstream effects, a variety of inflammatory syndromes known as irAEs can occur. Rheumatic irAEs include inflammatory arthritis, sicca syndrome, vasculitis, myositis and 
scleroderma [4, 5]. Inflammatory arthritis appears to be the most common rheumatic irAE, with prevalence estimates of $5 \%$ or higher [6].

Although inflammatory arthritis is a fairly common side effect of $\mathrm{ICl}$ therapy, the majority of treated patients do not develop this adverse event. Also, a wide range of tumour types in patients with ICl-induced inflammatory arthritis have been reported [7]. This raises the intriguing hypothesis that a predisposing genetic factor may influence which patients develop inflammatory arthritis due to $\mathrm{ICI}$ treatment.

$\mathrm{ICl}$-induced inflammatory arthritis shares some important similarities to classic forms of inflammatory arthritis. Patients can have small joint polyarthritis, similar to RA, and can have early erosive disease [8]. There are key differences, however, that suggest a separate immune pathogenesis from RA. First, patients are not predominantly female. Second, most patients lack traditional autoantibodies, RF and anti-CCPs. Additionally, patients may have features more consistent with spondyloarthritis such as enthesitis, reactive arthritis with conjunctivitis and urethritis, or inflammatory back pain [7].

In this pilot study, we aimed to evaluate whether class I and II HLA alleles that are associated with classic forms of inflammatory arthritis were more prevalent in patients with ICl-induced inflammatory arthritis when compared with healthy population controls. We also explored other potential HLA associations with complete highresolution typing of the HLA-A, -B, -C, -DRB1 and -DQB1 loci.

\section{Methods}

\section{Inclusion and exclusion criteria}

Patients over the age of 18 with rheumatologist-confirmed $\mathrm{ICl}$-induced inflammatory arthritis were included in the study if they had a DNA sample available for analysis. Patients with preexisting systemic autoimmune disease before $\mathrm{ICl}$ treatment were excluded.

Healthy controls were identified from potential bone marrow donors of European descent who had high resolution HLA typing performed at our institution.

Patients with physician-diagnosed RA (also of European descent) were drawn from a longitudinal cohort of patients followed for research at our institution and were included if they had a DNA sample available for analysis.

\section{Demographic and clinical data}

Information on demographics, oncologic history, ICI treatment and autoantibodies is collected as a part of an ongoing prospective cohort study of patients with $\mathrm{ICl}-$ induced inflammatory arthritis. This study was approved by the Johns Hopkins Institutional Review Board (IRB \# 00035182) and patients signed informed consent in order to participate.

\section{HLA typing}

High resolution HLA typing was performed at the CLIAcertified Immunogenetics Laboratory at Johns Hopkins
University by next generation sequencing using the TruSight HLA Sequencing Panel and the MiSeq System (Illumina, San Diego, CA). HLA types were assigned using the Assign TruSight HLA Analysis software. HLADRB1 ${ }^{\star} 01:$ 01, 01: 02, 04: 01, 04: 04, 04: 05, 04: 08, 10: 01, 14: 02 were designated as SE alleles $[9,10]$.

\section{Statistical analysis}

The frequency of having at least one SE allele was compared between the $\mathrm{ICl}$-induced inflammatory arthritis group and healthy controls and between the $\mathrm{ICl}$ induced inflammatory arthritis group and RA cases. The statistical assessment was made on Stata v.14 (StataCorp LLC, College Station, TX, USA) using Fisher's exact test. $P$ values, odds ratios $(\mathrm{OR})$ and $95 \%$ Cls were also obtained. At each of the HLA-A, -B, -C, -DRB1 and -DQB1 loci, allele-positivity rates for the four-digit HLA alleles were also compared between cases and controls. Only the top 25 most frequent alleles at each locus were compared, and the rest were grouped together for analysis. Given the exploratory nature of this study, no adjustment for multiple comparison was applied.

\section{Results}

Demographics, oncologic history and clinical features of $\mathrm{ICl}$-induced inflammatory arthritis

Twenty-seven patients with $\mathrm{ICl}$-induced inflammatory arthritis were included. The average age was 60.2 (s.D. 12.1) years, and 12 patients $(44.4 \%)$ were female. Melanoma was the most common tumour type $(n=9)$, followed by non-small cell lung cancer $(n=6)$. Other tumour types were renal cell carcinoma, basal cell carcinoma, squamous cell carcinoma, Merkel cell carcinoma, colon cancer, endometrial cancer, esophageal cancer, breast cancer and mycosis fungoides (Supplementary Table S1, available at Rheumatology online). Twenty-six patients were of self-identified European descent, and one was African American. Nine patients were treated with anti-PD-1/CTLA-4 combination therapy, and 18 patients were treated with anti-PD-1 or PD-L1 monotherapy (with or without an investigational agent, e.g. anti-CD73, anti-LAG3). Most patients lacked autoantibodies traditionally seen in RA; two were positive for RF (7.4\%) and two were positive for anti-CCP antibodies (7.4\%).

\section{HLA allele frequencies compared with population} controls

For this analysis, only the 26 patients of European descent were included. First, SE alleles were grouped together and the probability of having at least one SE allele was compared. In ICl-induced inflammatory arthritis, 16 (61.5\%) had at least one SE allele, while 299 (41.2\%) of the population control group had at least one SE allele (OR 2.3, $P=0.04$ ). Next, class I and II alleles known to be associated with traditional forms of inflammatory arthritis (i.e. RA, psoriatic arthritis, ankylosing spondylitis) were evaluated. These included the DRB1 SE alleles (analysed individually), $\mathrm{B}^{\star} 08$ : 01, $\mathrm{B}^{\star} 15$ : 01, $\mathrm{B}^{\star} 27$ : 05, $\mathrm{C}^{\star} 06$ : 02 and 
TABLE 1 Comparison of allele-positivity rates and SE alleles in ICl-induced inflammatory arthritis vs population controls of European descent

\begin{tabular}{|c|c|c|}
\hline HLA allele/s & $\begin{array}{l}\text { Odds ratio }(95 \% \mathrm{Cl}) \\
\text { ICl-induced } \\
\text { inflammatory } \\
\text { arthritis vs controls }\end{array}$ & $P$ value* \\
\hline$A^{\star} 03: 01$ & $2.2(0.9,5.1)$ & 0.07 \\
\hline$B^{*} 08: 01$ & $0.9(0.3,2.6)$ & 0.56 \\
\hline$B^{\star} 15: 01$ & $2.2(0.7,5.9)$ & 0.12 \\
\hline$B^{\star 2} 27: 05$ & $0.6(0.0,4.0)$ & 1.00 \\
\hline$B^{\star} 52: 01$ & $5.0(0.5,24.1)$ & 0.08 \\
\hline$C^{\star} 06: 02$ & $0.9(0.3,2.7)$ & 1.00 \\
\hline$C^{*} 12: 02$ & $5.4(0.6,26.8)$ & 0.07 \\
\hline DQB1*03: 01 & $0.4(0.1,1.1)$ & 0.06 \\
\hline DRB1*03: 01 & $1.1(0.4,2.9)$ & 0.81 \\
\hline DRB1*04: 05 & $8.6(1.7,43.4)$ & 0.04 \\
\hline At least $1 \mathrm{SE}$ allele & $2.3(1.0,5.1)$ & 0.04 \\
\hline
\end{tabular}

${ }^{*} P$ values $<0.05$ significant (bold). SE: shared epitope; ICl: immune checkpoint inhibitor.

DRB1*03: 01 [11-13]. When individual alleles were examined for associations, some showed frequency deviations in cases with $\mathrm{ICl}$-induced inflammatory arthritis (Table 1). HLA DRB1*04: 05 was enriched in the ICl-induced inflammatory arthritis population (OR 8.6, $P=0.04$ ). Also included in Table 2 are associations for other individual HLA alleles not increased in traditional forms of inflammatory arthritis, but with $P$ values approaching statistical significance (Table 1). There were trends toward higher prevalence of HLA A*03: 01 (OR 2.2, $P=0.07$ ), HLA $B^{\star} 52$ : 01(OR 5.0, $P=0.08$ ) and HLA C*12: 02 (OR 5.2, $P=0.07)$ in $\mathrm{ICl}$-induced inflammatory arthritis. Patients with $\mathrm{ICl}$-induced inflammatory arthritis had a trend toward lower prevalence of $\mathrm{DQB} 1{ }^{\star} 03$ : 01 (OR 0.4, $P=0.06$ ).

Within the group of $\mathrm{ICl}$-induced inflammatory arthritis patients, we evaluated whether clinical characteristics differed between patients with and without SE allele. Interestingly, some clinical features were only seen in patients lacking SE alleles. Specifically, inflammatory back pain, trigger fingers, prominent enthesitis and the case of reactive arthritis were seen in patients without SE alleles. None of these features were seen in the SE positive group. Small joint involvement including the small joints of hands and wrists, typically thought of as RA-like, was seen in those with and without SE alleles.

\section{Comparison of $\mathrm{ICl}$-induced inflammatory arthritis to RA population}

The probability of having at least one SE allele did not differ between patients of European descent with IClinduced inflammatory arthritis and RA patients of European descent (Table 2). There was a trend toward the RA patients being more likely to be homozygous for SE alleles, with $23.6 \%$ of RA cases being homozygous vs just $7.7 \%$ of $\mathrm{ICl}$-induced inflammatory arthritis $(P=0.15)$.
There were significant differences in RF and anti-CCP positivity (Table 2). There were no patients with IClinduced inflammatory arthritis who were positive for both RF and anti-CCP antibodies, as compared with the RA population, where $49.3 \%$ were double positive.

\section{Discussion}

In this study of high resolution HLA typing in ICl-induced inflammatory arthritis, patients of European descent were more likely to be positive for SE alleles than population controls. There were also suggestions of other HLA allelic associations with $\mathrm{ICl}$-induced inflammatory arthritis. There was not a significant difference in prevalence of SE between patients with ICl-induced inflammatory arthritis and those with RA of European descent. As previously shown, patients with ICl-induced inflammatory arthritis were unlikely to have RF or anti-CCP antibodies [7, 14, 15], and this was significantly different from the RA comparator population.

This is one of the first studies to evaluate immunogenetics in patients who develop an irAE due to ICl therapy. A smaller study of acute onset type 1 diabetes due to antiPD-1 therapy showed four out of five patients had HLA subgroups DRB01* 03 or 04, which are associated with type 1 diabetes risk in the general population [16]. Another study of patients with metastatic melanoma treated with ipilimumab suggests that having CTLA-4 gene variant $1661 \mathrm{~A}>\mathrm{G}$ may predispose patients to developing endocrine irAEs [17]. These data evaluated with the present study suggest that a patient's immunogenetic framework may increase the risk for developing irAEs like inflammatory arthritis due to $\mathrm{ICl}$ therapy. This pilot study is hypothesis-generating for future genetic studies in irAEs. That SE appears to be enriched in this population was a surprising finding, given this population is mostly anti-CCP negative. Two patients with RA-like ICl-induced inflammatory arthritis have been previously reported to have HLA-DRB1 ${ }^{*} 01$ : 01, one of whom was positive for anti-CCP [18]. While seronegative RA has been found to be associated with SE [19], potential mechanisms underlying this are less clear. The trends observed toward higher prevalence of several class I alleles are also intriguing given associations of various class I alleles with ankylosing spondylitis, peripheral spondyloarthritis and psoriatic arthritis. Certain clinical features in subsets of patients with ICl-induced inflammatory arthritis such as enthesitis, reactive arthritis-like presentation, and inflammatory back pain are seen in spondyloarthritis. Interestingly, there was no association with HLA B27, the allele most strongly associated with ankylosing spondylitis. This may suggest more similarity with peripheral spondyloarthritis or psoriatic arthritis in pathogenesis, rather than ankylosing spondylitis.

Our study also found that HLA B*52: 01 and $C^{*} 12$ : 02 may be associated with $\mathrm{ICl}$-induced inflammatory arthritis. Interestingly, in the Japanese population, the HLA-B ${ }^{\star} 52$ : 01-C*12: 02 haploptype has been associated with ulcerative colitis and Takayasu's arteritis [20] and HLA-C*12: 02 has been associated with late-onset psoriasis [21], 
TABLE 2 SE in European descent subset of ICl-induced inflammatory arthritis compared with ethnically matched RA controls

ICI-induced inflammatory arthritis $n=26(\%) \quad$ RA $n=220(\%)$

\begin{tabular}{llll} 
Positive for SE (at least 1 allele) & $16(61.5 \%)$ & $145(65.9 \%)$ & 0.66 \\
Number of SE alleles & Two alleles: $2(7.7 \%)$ & Two alleles: $52(23.6 \%)$ & 0.15 \\
& One allele: $14(53.8 \%)$ & One allele: $93(42.3 \%)$ & Zero allele: $75(34 \%)$ \\
& Zero allele: $10(38.5 \%)$ & $142(64.6 \%)$ & $<\mathbf{0 . 0 1}$ \\
CCP positive & $2(7.7 \%)$ & $122 / 215(56.7 \%)$ & $<\mathbf{0 . 0 1}$ \\
RF positive & $2(7.7 \%)$ & $106 / 215(49.3 \%)$ & $<\mathbf{0 . 0 1}$ \\
RF and CCP double positive & $0(0 \%)$ & $101 \%$ \\
\hline
\end{tabular}

*Fisher's exact test. $P$ values $<0.05$ significant (bold). CCP: anti-CCP antibodies; SE: shared epitope; ICl: immune checkpoint inhibitor.

suggesting potential relevance of these alleles to the development of autoimmune disease.

With increasing evidence that tumour response to ICls is improved in patients who develop irAEs [22-24], there may be shared genetic associations between response and toxicity. HLA typing and response to ICls was recently evaluated in a large study of patients with melanoma and non-small cell lung cancer; both patient and tumour HLA class I genotypes influenced tumour response to ICI therapy [25]. Specifically, patients with the HLA-B44 supertype had extended survival, while HLA-B62 supertype was associated with worse outcomes. Further genetic investigations could evaluate tumour response and irAE development concurrently.

The study was limited primarily by sample size, which was due to the naturally rare occurrence of $\mathrm{ICl}$-induced inflammatory arthritis at a single institution. As a result, smaller effect sizes were unlikely to be detected. Also, the cases of $\mathrm{ICl}$-induced inflammatory arthritis were not entirely clinically homogeneous, which could lower the statistical power of detecting an association. When a larger sample is available, it may be useful to look at groups by pattern of joint involvement or other clinical features to see if differing associations exist by subgroup (e.g. whether those with enthesitis have differing HLA associations from those with small joint polyarthritis or whether those with multiple irAEs have differing HLA associations vs patients with ICl-induced inflammatory arthritis alone). It will also be informative to study HLA associations in a broader group of $\mathrm{ICl}$-treated patients to define whether these alleles are risk factors for $\mathrm{ICl}$ induced inflammatory arthritis or other types of irAEs.

In summary, we observed a potential risk association between the presence of SE alleles and ICl-induced inflammatory arthritis in this pilot study of high resolution HLA typing. Larger studies are needed to confirm this association and to examine whether distinct HLA alleles associate with unique phenotypes of $\mathrm{ICl}$-induced inflammatory arthritis.

Funding: This work was supported by the National Institute of Arthritis, Musculoskeletal and Skin Disorders (P30AR070254, K23-AR061439, R01-AR073208), Jerome L.
Greene Foundation, the Passano Foundation, the Camille Julia Morgan Arthritis Research and Education Fund, Stabler Foundation Discovery Fund, The Scherr Family Foundation and Joanne and Adam Rogers, and an investigator-initiated study funded by Bristol-Myers Squibb.

Disclosure statement: L.C.C. has received research funding from Bristol-Myers Squibb. C.O.B. has received research funding from and served as a consultant for Bristol-Myers Squibb. A.A.S. has served as a consultant for Bristol-Myers Squibb.

\section{Supplementary data}

Supplementary data are available at Rheumatology online.

\section{References}

1 Wahren-Herlenius M, Dorner T. Immunopathogenic mechanisms of systemic autoimmune disease. Lancet (Lond Engl) 2013;382:819-31.

2 Nguyen H, James EA. Immune recognition of citrullinated epitopes. Immunology 2016;149:131-8.

3 Topalian SL, Drake CG, Pardoll DM. Immune checkpoint blockade: a common denominator approach to cancer therapy. Cancer Cell 2015;27:450-61.

4 Barbosa NS, Wetter DA, Wieland CN et al. Scleroderma induced by pembrolizumab: a case series. Mayo Clin Proc 2017;92:1158-63.

5 Cappelli LC, Gutierrez AK, Bingham CO 3rd, Shah AA. Rheumatic and musculoskeletal immune-related adverse events due to immune checkpoint inhibitors: a systematic review of the literature. Arthritis Care Res 2017;69:1751-63.

6 Buder-Bakhaya K, Benesova K, Schulz C et al. Characterization of arthralgia induced by PD-1 antibody treatment in patients with metastasized cutaneous malignancies. Cancer Immunol Immunother 2018;67:175-82.

7 Cappelli LC, Brahmer JR, Forde PM et al. Clinical presentation of immune checkpoint inhibitor-induced inflammatory arthritis differs by immunotherapy regimen. Semin Arthritis Rheum 2018;doi:10.1016/j.semarthrit.2018.02.011. 
8 Cappelli LC, Gutierrez AK, Baer AN et al. Inflammatory arthritis and sicca syndrome induced by nivolumab and ipilimumab. Ann Rheum Dis 2017;76:43-50.

9 Balsa A, Cabezón A, Orozco G et al. Influence of HLA DRB1 alleles in the susceptibility of rheumatoid arthritis and the regulation of antibodies against citrullinated proteins and rheumatoid factor. Arthritis Res Ther 2010;12:R62.

10 van der Woude D, Lie BA, Lundström E et al. Protection against anti-citrullinated protein antibody-positive rheumatoid arthritis is predominantly associated with HLA-DRB1*1301: a meta-analysis of HLA-DRB1 associations with anti-citrullinated protein antibody-positive and anti-citrullinated protein antibody-negative rheumatoid arthritis in four European populations. Arthritis Rheum 2010;62:1236-45.

11 van Drongelen V, Holoshitz J. Human leukocyte antigendisease associations in rheumatoid arthritis. Rheum Dis Clin North Am 2017;43:363-76.

12 Santos AM, Peña P, Avila M et al. Association of human leukocyte $A, B$, and $D R$ antigens in Colombian patients with diagnosis of spondyloarthritis. Clin Rheumatol 2017;36:953-8.

13 Winchester R, Giles J, Jadon D et al. Implications of the diversity of class I HLA associations in psoriatic arthritis. Clin Immunol 2016;172:29-33.

14 Calabrese C, Kirchner E, Kontzias K, Velcheti V, Calabrese LH. Rheumatic immune-related adverse events of checkpoint therapy for cancer: case series of a new nosological entity. RMD Open 2017;3:e000412.

15 Smith MH, Bass AR. Arthritis after cancer immunotherapy: symptom duration and treatment response. Arthritis Care Res 2017; doi:10.1002/acr.23467.

16 Magis Q, Gaudy-Marqueste C, Basire A et al. Diabetes and blood glucose disorders under anti-PD1. J Immunother 2018;41:232-40.
17 Queirolo P, Dozin B, Morabito A et al. CTLA-4 gene variant $-1661 A>G$ may predict the onset of endocrine adverse events in metastatic melanoma patients treated with ipilimumab. Eur J Cancer 2018;97:59-61.

18 Kostine M, Rouxel L, Barnetche T et al. Rheumatic disorders associated with immune checkpoint inhibitors in patients with cancer-clinical aspects and relationship with tumour response: a single-centre prospective cohort study. Ann Rheum Dis 2018;77:393-8.

19 van der Woude D, Young A, Jayakumar K et al. Prevalence of and predictive factors for sustained disease-modifying antirheumatic drug-free remission in rheumatoid arthritis: results from two large early arthritis cohorts. Arthritis Rheum 2009;60:2262-71.

20 Terao C, Matsumura T, Yoshifuji $\mathrm{H}$ et al. Takayasu arteritis and ulcerative colitis: high rate of co-occurrence and genetic overlap. Arthritis Rheumatol 2015;67:2226-32.

21 Mabuchi T, Ota T, Manabe $\mathrm{Y}$ et al. HLA-C ${ }^{\star} 12$ : 02 is a susceptibility factor in late-onset type of psoriasis in Japanese. J Dermatol 2014;41:697-704.

22 Judd J, Zibelman M, Handorf E et al. Immune-related adverse events as a biomarker in non-melanoma patients treated with programmed cell death 1 inhibitors. Oncologist 2017;22:1232-7.

23 Downey SG, Klapper JA, Smith FO et al. Prognostic factors related to clinical response in patients with metastatic melanoma treated by CTL-associated antigen- 4 blockade. Clin Cancer Res 2007;13:6681-8.

24 Haratani K, Hayashi H, Chiba $\mathrm{Y}$ et al. Association of immune-related adverse events with nivolumab efficacy in non-small-cell lung cancer. JAMA Oncol 2018;4:374-8.

25 Chowell D, Morris LGT, Grigg CM et al. Patient HLA class I genotype influences cancer response to checkpoint blockade immunotherapy. Science 2018;359:582-7. 